\title{
Iron Overload Cardiomyopathy due to Non-Classical Hereditary Hemochromatosis
}

\author{
Ali Hamoudia, c, Vian Taqi ${ }^{b}$
}

\begin{abstract}
Hemochromatosis is defined as abnormal deposition of iron in body organs which can cause organ dysfunction. The most commonly affected organs are the liver, heart, endocrine glands, pancreas, joints and skin with manifestations of cirrhosis, cardiomyopathy, hypogonadism, endocrinopathies, diabetes mellitus, arthropathy, skin pigmentation, and increased risk of liver cancer in cirrhotic patients. The disease severity ranges from asymptomatic tissue damage with simple biochemical abnormalities detected incidentally to serious life-threatening disease that usually affects young people. Excessive iron accumulation in the body usually takes place either by genetic mutations causing increased gastrointestinal iron absorption (hemochromatosis) or secondary to excessive administration of exogenous iron by red blood cell transfusions or dietary sources (hemosiderosis). Cardiac hemochromatosis or primary iron-overload cardiomyopathy is recognized as the presence of systolic or diastolic cardiac dysfunction secondary to increased deposition of iron in the heart in the absence of other concomitant processes. Although heart failure secondary to iron overload could be lethal, it is potentially a preventable and a treatable disease when diagnosed and treated early in its course.
\end{abstract}

Keywords: Hemochromatosis; Phlebotomy; Cardiomyopathy; Atrial fibrillation

\section{Introduction}

Cardiac hemochromatosis is a rare and serious medical condition that is caused by excessive iron deposition in the heart. Although it may be presented late in women due to menstrual blood loss, men usually present with it earlier in the third or fourth decade of life with symptoms of heart failure or arrhythmia. This is a case of a young patient presenting with heart failure symptoms with incidental elevated iron level in the body in the absence of common risk factors of heart disease.

Manuscript submitted January 7, 2019, accepted January 18, 2019

aRosalind Franklin University, North Chicago, IL, USA

bUniversity of Baghdad, Baghdad, Iraq

${ }^{\mathrm{c}}$ Corresponding Author: Ali Hamoudi, Rosalind Franklin University, North

Chicago, IL 60064, USA. Email: ali.hamoudi@rosalindfranklin.edu

doi: https://doi.org/10.14740/jmc3240

\section{Case Report}

We present a case of a previously healthy 32-year-old Hispanic man who presented with newly onset shortness of breath, orthopnea, intermittent palpitation and bilateral leg swelling and unintentional 10 pounds weight gain during the last month. He reported that his shortness of breath is progressive and he could not walk more than one block recently. He denied any recent chest pain, dizziness, syncope, fever, night sweats, recent hospitalization or travel history. The patient was recently diagnosed with insulin-dependent diabetes mellitus and was also told that he has abnormal liver functions when he received abdominal ultrasound for other reasons although he denied any history of alcohol, tobacco or illicit drug use.

Upon hospital admission, blood pressure (BP) was 96/68, pulse rate 103 , respiratory rate 18 , and oral temperature 97.6 ${ }^{\circ} \mathrm{F}\left(36.4{ }^{\circ} \mathrm{C}\right)$ with $\mathrm{SpO}_{2}$ of $97 \%$ and body mass index (BMI) of $25.50 \mathrm{~kg} / \mathrm{m}^{2}$.

On examination jugular venous distention was noticed with heart examination showing irregular heartbeats, normal $\mathrm{S} 1$ and S2, without murmurs or rub. Bilateral lungs crackles in the mid and lower lungs were heard in addition to bilateral leg pitting edema. He had normal hemoglobin level, mild elevated liver function test (LFT), and elevated brain natriuretic peptide (BNP) of 1,517. Iron panel showed a high iron level of 255 and low unsaturated iron-binding capacity (UIBC) of $<55$, and transferrin of 214 with elevated ferritin of 7,049. Common hemochromatosis gene mutations (C282Y, H63D and S65C) were negative.

Chest X-ray showed pulmonary congestions with bilateral pleural effusions. Electrocardiogram (EKG) showed new onset atrial fibrillation (A-fib) with transthoracic echocardiogram showing mild left ventricular hypertrophy with newly reduced left ventricular ejection fraction (EF) (30-35\%) in addition to diastolic dysfunction. Abdominal magnetic resonance imaging (MRI) showed hepatic hemochromatosis with secondary cirrhosis and portal hypertension. The patient was diagnosed with acutely decompensated heart failure secondary to hemochromatosis that was confirmed with cardiac MRI. Our patient was treated for heart failure and A-fib and started on weekly phlebotomy. His condition was improved significantly afterward.

\section{Discussion}

The cornerstone of diagnosis of hemochromatosis relies on 
Table 1. Primary Hereditary Hemochromatosis due to Increased Gastrointestinal (GI) Absorption of Normal Diet

\begin{tabular}{lll}
\hline Diseases & Molecular correlates & Organs affected \\
\hline $\begin{array}{l}\text { Type 1 (HFE-related) (AR), } \\
\text { the most common type }\end{array}$ & $\begin{array}{l}\text { Missense mutation in C282Y homozygosity, H63D homozygosity, } \\
\text { C282Y/H63D heterozygosity, and other mutations of HFE }\end{array}$ & Liver, heart, and endocrine glands \\
$\begin{array}{l}\text { Type 2 (juvenile } \\
\text { hemochromatosis) (AR) }\end{array}$ & $\begin{array}{l}\text { Type 2 A Hemojuvelin protein (HJV gene), Type 2 B Hepcidin } \\
\text { protein (HAMP gene) }\end{array}$ & $\begin{array}{l}\text { Liver, heart, endocrine glands, and } \\
\text { skeletal muscles }\end{array}$ \\
Type 3 (AR) & Mutation in transferrin receptor 2 & Liver, heart, and endocrine glands \\
Type 4 (AD) & $\begin{array}{l}\text { Mutation of solute carrier family 40, member 1(SLC40A1) which } \\
\text { encodes for ferroportin }\end{array}$ & $\begin{array}{l}\text { Macrophages, liver, heart, and } \\
\text { endocrine glands }\end{array}$ \\
\hline
\end{tabular}

HJV: hemojuvelin-encoding gene; HLA: human leukocyte antigen; TFR1: transferrin receptor 1.

molecular diagnosis. The genetic heterogeneity of hemochromatosis is mediated by hepcidin-ferroportin interaction. Most cases of adult-onset hemochromatosis arise from single point mutation at position $282(\mathrm{C} 282 \mathrm{Y})$ of the hydration free energy (HFE) protein that results in the substitution of tyrosine for cysteine. It accounts for about $80 \%$ of hemochromatosis and is particularly common in northern Europe. It is also most common in whites, but extremely rare in nonwhites.

The second common mutation is characterized by the replacement of histidine by aspartic acid at position 63 of the protein (H63D). Its contribution to the clinical disease is minimal, except in compound heterozygosity with C282Y. Although other mutations were discovered, they are much less common as those below. Our patient, however, presented with C282Y and H63D negative mutations. Table 1 shows the most common etiology of iron overload in hemochromatosis $[1,2]$.

Secondary hemochromatosis includes that secondary to iron-loading anemia (transfusion-related), such as in thalassemia, sickle cell anemia, sideroblastic anemia, diamond blackfan anemia, congenital dyserythropoesis anemia, chronic renal disease, end-stage renal disease, dialysis and post-stem cell transplant patients, and that secondary to dietary overload (African iron overload), due to increased diet with genetic predisposition.

The miscellaneous type includes fatty liver disease, chronic liver disease, hepatitis B and C, alcoholic liver disease, porphyria cutanea tarda, and aceruloplasminemia.

Pathologic iron deposition begins initially within the epicardium and extends towards the myocardium and then endocardium, which helps explain the preservation of heart systolic function until very late in the disease [3]. Myocardial cell damage in hemochromatosis is mediated by production of reactive free radicals especially hydroxyl ions [4].

The initial symptoms are quite variable ranging from totally asymptomatic early in the disease to terminal irreversible heart failure symptoms in severely iron overloaded patients.

Patients often presented with exertional shortness of breath (as in our patient) as a result of left ventricular diastolic dysfunction secondary to restrictive pathophysiology. As the condition progresses to a dilated cardiomyopathy, left ventricular systolic failure can happen and more symptoms develop [5].

Iron deposition in the conduction system can cause different arrhythmias with paroxysmal A-fib being the most common arrhythmia seen in iron-induced cardiomyopathy [6].

As the extent of iron deposition increases in atrium, su- praventricular arrhythmias and first-degree atrioventricular blocks may happen, which correlates with the extent of disease [7]. Left ventricular dilatation with systolic dysfunction predisposes to more frequent ventricular arrhythmias. As severe cardiac impairment happens, average survival is usually less than 1 year [8].

Hemochromatosis should be suspected due to positive family history, abnormal hepatic enzymes, endocrinopathies, or other organ involvement which are followed by measurements of ferritin level and transferrin saturation. A transferrin saturation of greater than $55 \%$ and a serum ferritin concentration of greater than $300 \mu \mathrm{g} / \mathrm{L}$ in men and greater than 200 $\mu \mathrm{g} / \mathrm{L}$ in women make the diagnosis of hemochromatosis after exclusion of secondary causes of iron overload. Diagnosis of hemochromatosis nowadays relies on genetic testing. Testing for HFE and H63D mutations should be performed once iron overload conformed to differentiating hereditary hemochromatosis from hemosiderosis. Those patients who test positive for $\mathrm{C} 282 \mathrm{Y}$ homozygote and the C282Y/H63D compound heterozygote are considered at highest risk for the disease.

As iron overload proceeds, echocardiography may reveal ventricular diastolic dysfunction with cardiac dilatation and progressive evidence of restrictive cardiomyopathy from myocardial damage [9].

Iron deposition in the heart tends to be patchy; therefore biopsies may miss the areas of deposition and provide a false negative result. Quantitation of liver iron can be obtained by measuring liver iron concentration (LIC) in the liver, which can be noninvasively measured using superconducting quantum interference device (SQUID), a very sensitive technique, but its use is limited by the very small number of available machines [10].

Alternatively, MRI is the only presently available noninvasive method with the potential to assess quantitatively myocardial iron load. It provides an accurate reproducible measure of left ventricular systolic EF that can be followed over the course of therapy. Non-iron overloaded hearts tend to be homogenous and relaxation time lasts for a longer duration. Hearts with iron overload, however, produce changes in MR signal intensity, which shortens the relaxation time and darkens the image more quickly. T2* method which stands for time constant decay of gradient echo in milliseconds is highly sensitive and specific for quantitation of myocardial iron deposition. As myocardial iron deposition increases, the relaxation 
time will be shortened. Thus, T2* will be shorter, and progressive decline in left ventricular EF will be noticed [11]. Cardiac T2* value is useful in providing guidance through the course of treatment and assessing the time interval for monitoring of cardiac function [12].

Management of hemochromatosis is mandatory especially in symptomatic patients once iron level increases significantly to protect the heart and prevent liver fibrosis. Phlebotomy remains the most effective treatment that prevents organ damage and increases survival if started early during disease. The recommended protocol is to remove (one or two) 400 - to $500-\mathrm{mL}$ units of blood (approximately 200-250 mg iron in each unit) weekly, until serum ferritin level is less than $50 \mathrm{ng} / \mathrm{mL}$ and transferrin saturation less than $30 \%$ thereafter by periodic removal of blood.

Once iron depletion is reached, lifelong therapy with three to four phlebotomies per year maintains the iron store at an adequately low level. Improvements in cardiac function in hemochromatosis patients with cardiomyopathy and refractory arrhythmias have been noted with aggressive iron removal with phlebotomy, especially when started early in the disease process. Gonadal dysfunction and insulin-dependent diabetes mellitus require lifelong hormonal replacement therapy.

At present, the only therapy alternative to phlebotomy for patients with anemia or severe cardiac disease is iron chelation to detoxify body organs. Patients can be managed with chelators like deferoxamine, deferasirox, and deferiprone with protocols that are similar to those established for secondary iron overload. Serum ferritin and urinary iron excretion are used to monitor the treatment efficacy. Erythropoietin has been used in selected patients to increase hemoglobin levels and to allow concomitant phlebotomy.

\section{Conclusions}

The goal of this case report is to consider the possibility of cardiac hemochromatosis in any patient with unexplained heart failure with elevated serum iron especially in the absence of significant risk factors. Cardiac hemochromatosis is a potentially lethal but preventable disease. Imaging using MRI in addition to genetic testing has replaced liver biopsy which is restricted to patients who do not have an informative genotype or is used for prognostic evaluation in cases of severe iron loading. Frequent iron studies in addition to phlebotomy form the cornerstone for disease management. This case shows a rare and interesting young patient who was tested negative for the common mutations. However, diagnosis of hemochromatosis was established based on clinical, biochemical and imaging studies. This was confirmed by significant improvement in patient's symptoms as well as his cardiac function with the most recent left ventricular EF being 54\% after phlebotomy was initiated.

\section{Conflict of Interest}

The authors declare that they have no conflict of interest.

\section{Financial Support}

No financial support or grants have been provided.

\section{Author Contributions}

Co-authors helped write or edit the manuscript. All authors read and approved the final manuscript.

\section{Abbreviations}

LFT: liver function test; BNP: brain natriuretic peptide; UIBC: unsaturated iron-binding capacity; MRI: magnetic resonance imaging; A-fib: atrial fibrillation; EF: ejection fraction; EKG: electrocardiogram; BP: blood pressure; BMI: body mass index; HFE: hydration free energy

\section{References}

1. Gujja P, Rosing DR, Tripodi DJ, Shizukuda Y. Iron overload cardiomyopathy: better understanding of an increasing disorder. J Am Coll Cardiol. 2010;56(13):1001-1012.

2. Roetto A, Totaro A, Piperno A, Piga A, Longo F, Garozzo G, Cali A, et al. New mutations inactivating transferrin receptor 2 in hemochromatosis type 3. Blood. 2001;97(9):2555-2560.

3. Liu P, Olivieri N. Iron overload cardiomyopathies: new insights into an old disease. Cardiovasc Drugs Ther. 1994;8(1):101-110.

4. Olson LJ, Edwards WD, McCall JT, Ilstrup DM, Gersh BJ. Cardiac iron deposition in idiopathic hemochromatosis: histologic and analytic assessment of 14 hearts from autopsy. J Am Coll Cardiol. 1987;10(6):12391243.

5. Unlusoy Aksu A, Caleffi A, Pietrangelo A, Sari S, Egritas Gurkan O, Demirtas Z, Yilmaz G, et al. Iron overload in the liver of 2 children: nonalcoholic steatohepatitis and juvenile hemochromatosis. J Pediatr Hematol Oncol. 2017;39(6):466-469.

6. Hahalis G, Alexopoulos D, Kremastinos DT, Zoumbos NC. Heart failure in beta-thalassemia syndromes: a decade of progress. Am J Med. 2005;118(9):957-967.

7. Buja LM, Roberts WC. Iron in the heart. Etiology and clinical significance. Am J Med. 1971;51(2):209-221.

8. Fitchett DH, Coltart DJ, Littler WA, Leyland MJ, Trueman T, Gozzard DI, Peters TJ. Cardiac involvement in secondary haemochromatosis: a catheter biopsy study and analysis of myocardium. Cardiovasc Res. 1980;14(12):719724.

9. Lubitz SA, Goldbarg SH, Mehta D. Sudden cardiac death in infiltrative cardiomyopathies: sarcoidosis, scleroderma, amyloidosis, hemachromatosis. Prog Cardiovasc Dis. 2008;51(1):58-73.

10. Nielsen P, Engelhardt R, Dullmann J, Fischer R. Non-invasive liver iron quantification by SQUID-biosusceptom- 
etry and serum ferritin iron as new diagnostic parameters in hereditary hemochromatosis. Blood Cells Mol Dis. 2002;29(3):451-458.

11. Anderson LJ, Holden S, Davis B, Prescott E, Charrier CC, Bunce NH, Firmin DN, et al. Cardiovascular T2-star
$(\mathrm{T} 2 *)$ magnetic resonance for the early diagnosis of myocardial iron overload. Eur Heart J. 2001;22(23):21712179.

12. Wood JC. Magnetic resonance imaging measurement of iron overload. Curr Opin Hematol. 2007;14(3):183-190. 